[Supporting Information]

\title{
Structure-Conductivity Relationships in Ordered and Disordered Salt-Doped Diblock Copolymer/Homopolymer Blends
}

\author{
Matthew T. Irwin, ${ }^{1}$ Robert J. Hickey, ${ }^{2}$ Shuyi Xie, ${ }^{2}$ Soonyong So, ${ }^{1}$ \\ Frank S. Bates, ${ }^{1}$ and Timothy P. Lodge ${ }^{*, 1,2}$ \\ ${ }^{1}$ Department of Chemical Engineering and Materials Science and ${ }^{2}$ Department of Chemistry, \\ University of Minnesota, Minneapolis, MN 55455
}

Authors for correspondence: lodge@umn.edu, bates001@umn.edu 

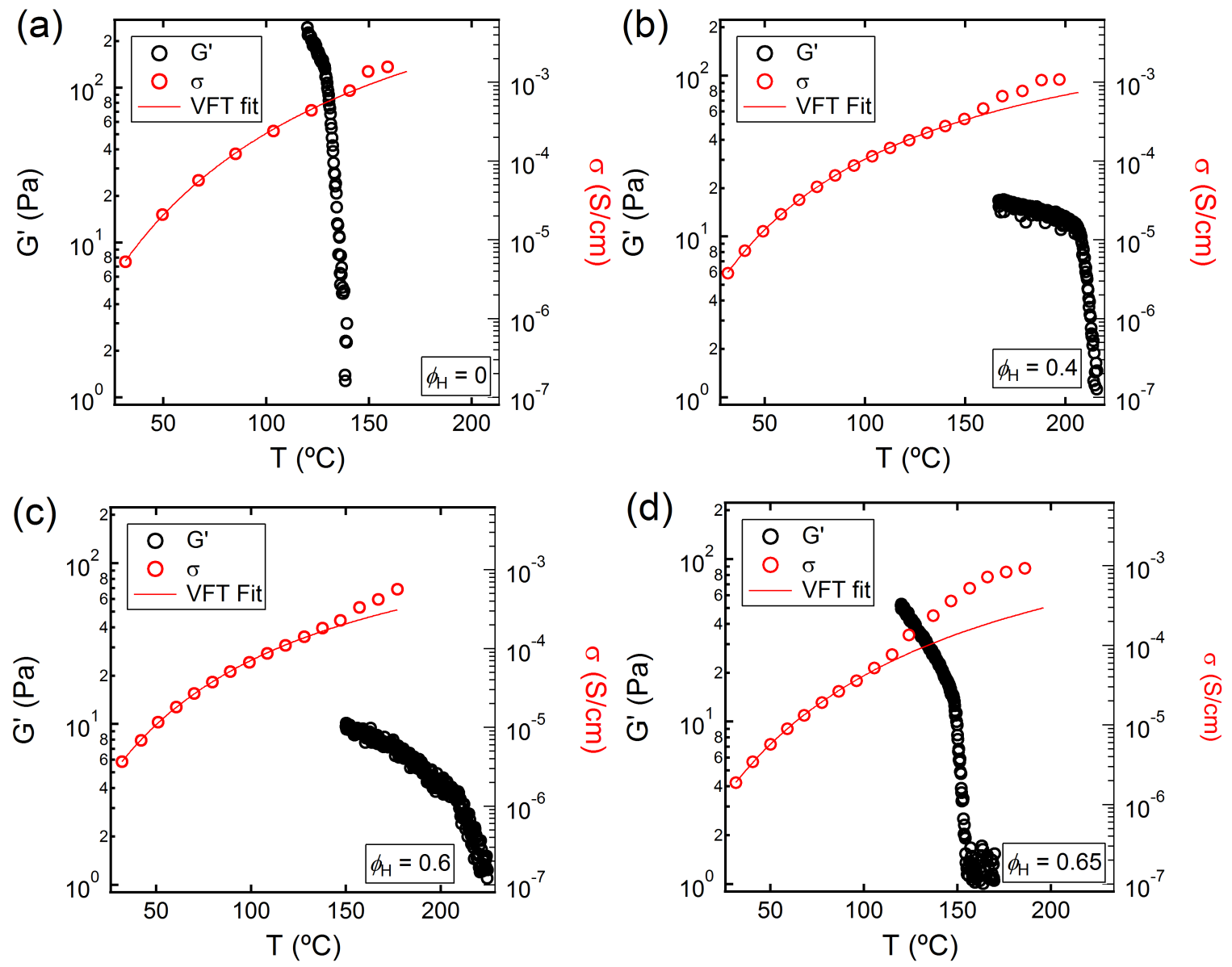

Figure S1. Representative elastic modulus and conductivity vs. temperature for blends containing homopolymer loadings, $\phi_{\mathrm{H}}$, of (a) 0 , (b) 0.4 , (c) 0.6 , and (d) 0.65 . 
Table S1. VFT fitting parameters used for fitting the ordered state conductivity data in Figures 4 and S4.

\begin{tabular}{cccc}
\hline $\boldsymbol{\phi}_{\mathbf{H}}$ & $\boldsymbol{\sigma}_{\mathbf{0}}(\mathbf{S} / \mathbf{c m})$ & $\boldsymbol{B}(\mathbf{K})$ & $\boldsymbol{T}_{\mathbf{0}}(\mathbf{K})$ \\
\hline 0 & 0.265 & 1400 & 175 \\
0.2 & 0.202 & 1360 & 169 \\
0.4 & 0.019 & 906 & 198 \\
0.6 & 0.010 & 906 & 191 \\
0.65 & 0.019 & 1252 & 169 \\
0.7 & 0.167 & 2600 & 115 \\
\hline
\end{tabular}

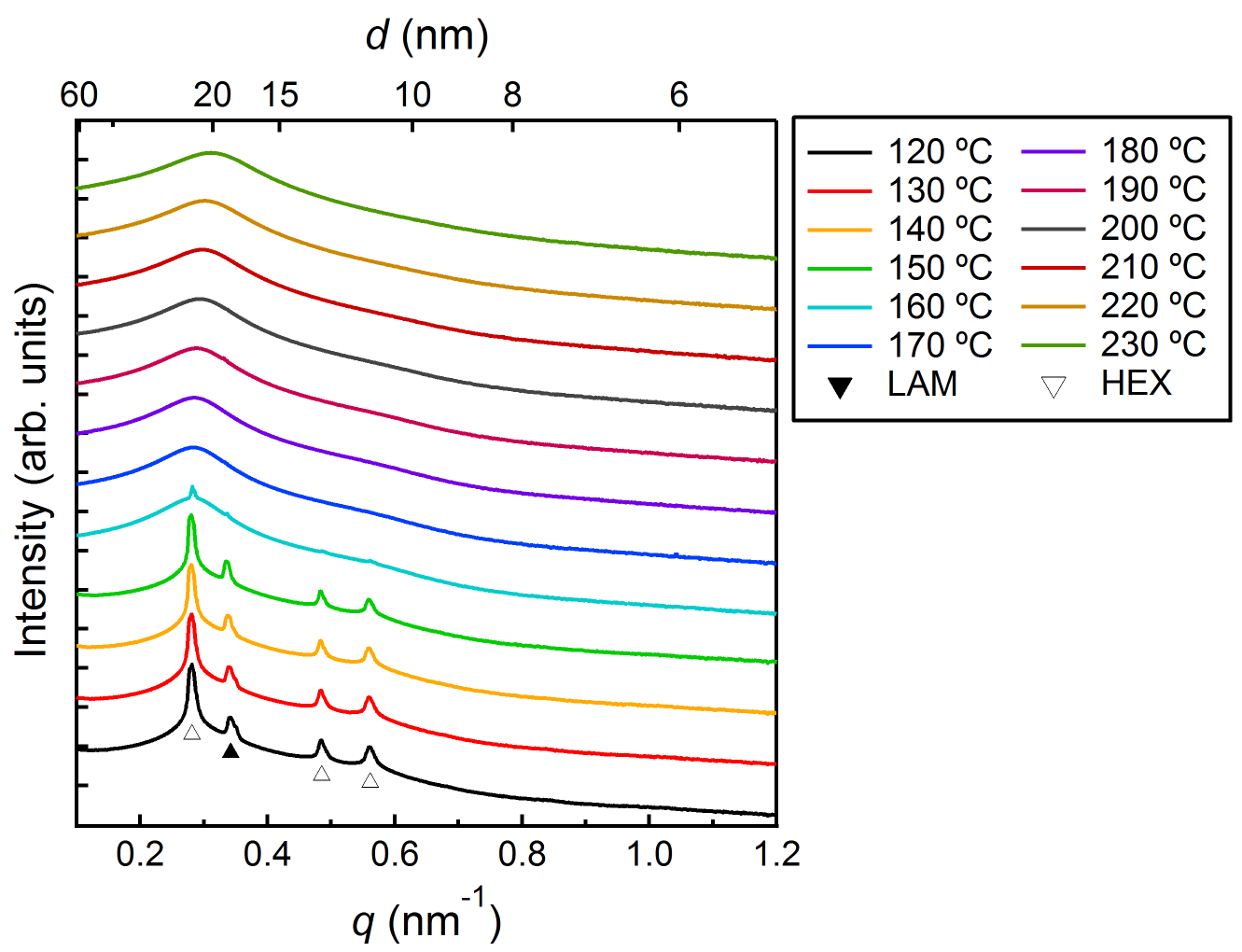

Figure S2. Small angle X-ray scattering traces for a blend containing $\phi_{\mathrm{H}}=0.7$ doped with LiTFSI at a molar ratio $r=\left[\mathrm{Li}^{+}\right] /[\mathrm{EO}]=0.06$. Reproduced from Ref. 1 . 
(a)

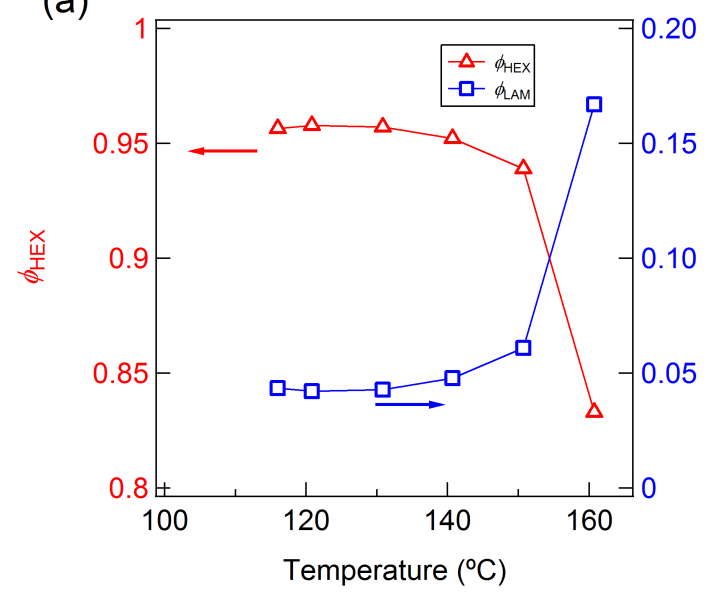

(b)

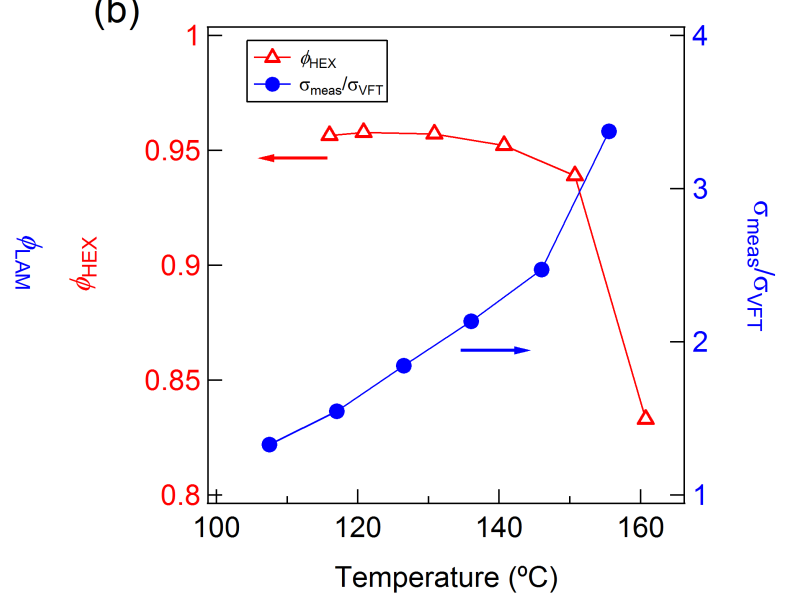

Figure S3. Analysis of ordered-state SAXS data from Figure S2 for a blend containing $\phi_{\mathrm{H}}=0.7$ doped with LiTFSI at a molar ratio $r=\left[\mathrm{Li}^{+}\right] /[\mathrm{EO}]=0.06$. Part (a) shows the relative fraction of each ordered phase as a function of temperature, while (b) shows that the ratio between the measured and extrapolated conductivity values increases as the fraction of HEX decreases. The relative fraction of each phase was determined by comparing the relative intensity of the primary scattering peak of each phase. Lines have been drawn to guide the eye. 


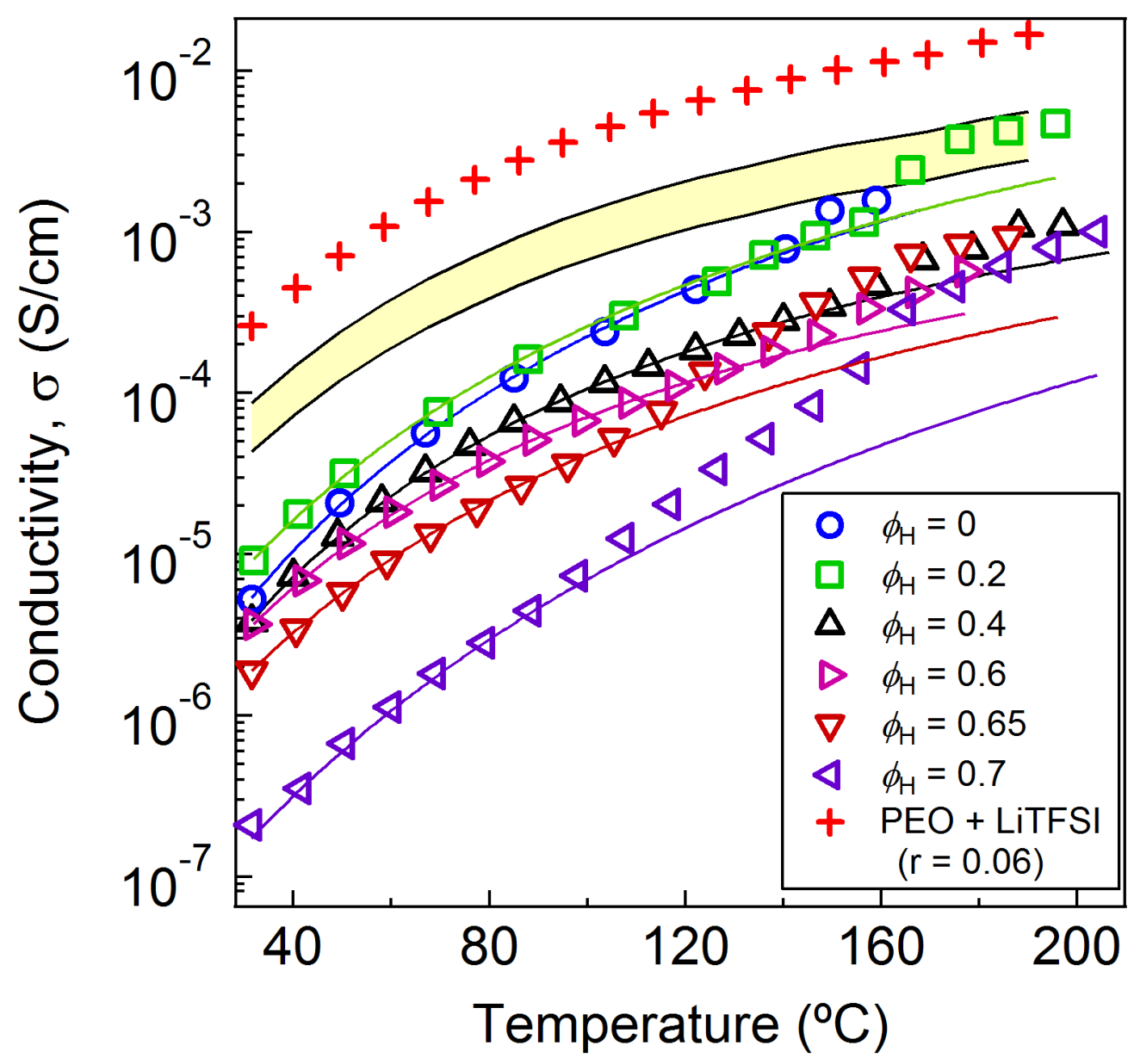

Figure S4. Comparison of conductivity for various blend compositions including data for $\phi_{\mathrm{H}}=$ 0.6 homopolymer blends. The lines through data represent VFT fits to the ordered-state data and help to visualize the large change in conductivity through the order-disorder transition. The red crosses represent the conductivity measured for a PEO homopolymer and LiTFSI blend with $r=$ 0.06 , and the shaded region below the crosses represent the conductivity adjusted for a range of blend morphologies according to Equation 1 in the main text. 

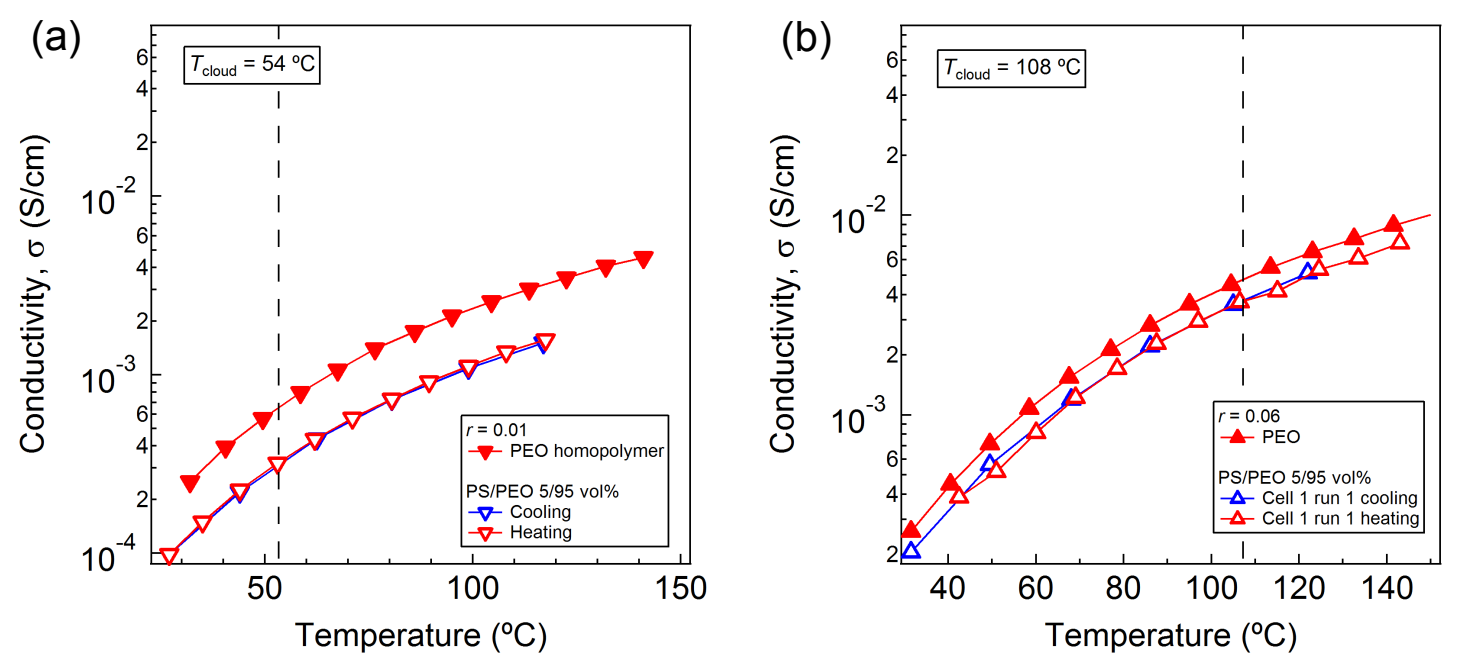

Figure S5. Ionic conductivity for blends that blends that contain PS/PEO homopolymers at 5/95 vol\% with $r=$ (a) 0.01 and (b) 0.06. Surprisingly, the temperature dependence of the conductivity of the blends does not deviate at the cloud point, potentially due to the lack of stirring within the coin cells to promote phase separation. Lines have been drawn to guide the eye.

\section{Pulsed-field gradient nuclear magnetic resonance (PFG-NMR)}

\section{Experimental}

PFG-NMR experiments were performed using a Bruker Advance III $500 \mathrm{MHz}$ NMR spectrometer. Samples were prepared using a double-capillary geometry in which the bulk sample was placed in the outer capillary, and DMSO-d6 was placed in the inner capillary. This geometry was necessary for locking and shimming, as the bulk samples did not contain any solvent. All experiments were carried out at $75^{\circ} \mathrm{C}$.

\section{Results and Discussion}

To better understand how the conductivity of both the lithium and TFSI ions change with blend composition, PFG-NMR experiments were conducted to determine the diffusion coefficients of PEO, $\mathrm{Li}^{+}$, and $\mathrm{TFSI}^{-}$in the salt-doped PEO homopolymer. The values for the diffusion coefficient of the three components in our LiTFSI-doped PEO homopolymers are compared against literature values for both other salt-doped PEO homopolymer systems and a PS-PEO block copolymer in Table S2 and Figure S6. 
The results in the table and figure indicate that the hydroxyl-terminated PEO homopolymer, $\mathrm{Li}^{+}$, and $\mathrm{TFSI}^{-}$in the current study diffuse approximately an order of magnitude slower than the same salt dissolved in the dimethoxy-terminated PEO homopolymer studied by Orädd et al. ${ }^{2}$ This result is likely due to significant hydrogen bonding in the hydroxyl-terminated PEO homopolymer that results in a higher viscosity and lower overall diffusion coefficients. Compared to the high molecular weight PEO hompolymer and salt-doped block copolymers, however, the diffusion coefficients for $\mathrm{Li}^{+}$and $\mathrm{TFSI}^{-}$in the hydroxyl-terminated PEO homopolymer are approximately $2-5$ times larger, indicating that the mobile PEO homopolymer still provides enhanced ion transport. These results suggest that blends that are relatively more block copolymer-rich should exhibit lower diffusion coefficients of $\mathrm{Li}^{+}$and $\mathrm{TFSI}^{-}$relative to the case of low molecular weight PEO homopolymer. Thus, our finding that blends with significantly more homopolymer have a lower conductivity (i.e., $\sigma_{\phi=0.7}<\sigma_{\phi=0}$ ) cannot be explained by a reduction in the diffusion coefficients of the ions.

Table S2. Diffusion coefficients of various species in various solvents.

\begin{tabular}{|c|c|c|c|c|c|c|}
\hline \multicolumn{7}{|c|}{ Diffusion Coefficients $\left(\mathrm{m}^{2} / \mathrm{s}\right)$} \\
\hline System & $\begin{array}{c}\boldsymbol{M}_{\mathbf{n}} \text { PEO } \\
(\mathrm{kg} / \mathrm{mol})\end{array}$ & $\boldsymbol{D}_{\mathrm{Li}}^{+}$ & $D_{\text {TFSI }}^{-}$ & $D_{\text {PEO }}$ & Method & Ref. \\
\hline $\begin{array}{c}\mathrm{HO}-\mathrm{PEO}-\mathrm{OH} \\
r=0.06,75^{\circ} \mathrm{C}\end{array}$ & 0.4 & $3.0 \times 10^{-11}$ & $4.3 \times 10^{-11}$ & $3.0 \times 10^{-11}$ & PFG-NMR & Our study \\
\hline $\begin{array}{c}\mathrm{H}_{3} \mathrm{CO}-\mathrm{PEO}-\mathrm{OCH}_{3} \\
r=0.05,85^{\circ} \mathrm{C}\end{array}$ & 0.4 & $1.1 \times 10^{-10}$ & $1.6 \times 10^{-10}$ & $1.3 \times 10^{-10}$ & PFG-NMR & 2 \\
\hline $\begin{array}{c}\text { PS-PEO } \\
r=0.085,90{ }^{\circ} \mathrm{C}\end{array}$ & $>20$ & $3.6 \times 10^{-12}$ & $1.4 \times 10^{-11}$ & - & $\begin{array}{c}\text { EIS, } \\
\text { assumes } \\
\mathrm{t}^{+}=0.2\end{array}$ & 3 \\
\hline
\end{tabular}




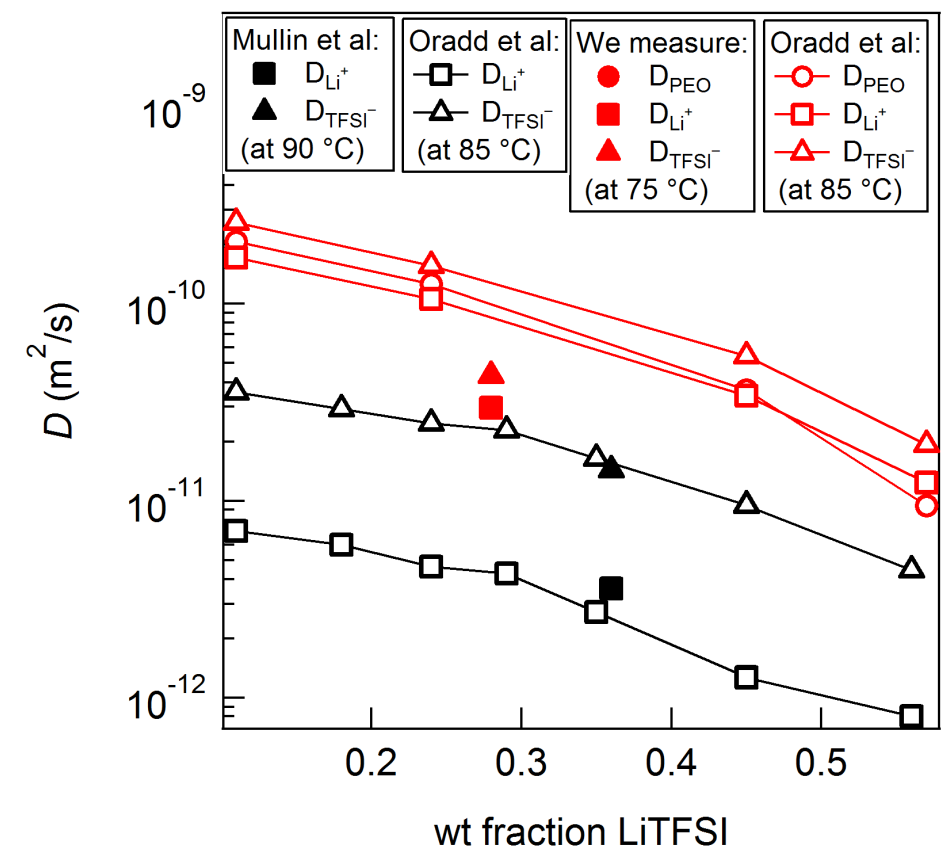

Figure S6. Diffusion coefficients of $\mathrm{PEO}, \mathrm{Li}^{+}$, and $\mathrm{TFSI}^{-}$in various environments and temperatures. The symbols in black refer to diffusion coefficients assessed in high molecular weight PEO $\left(M_{\mathrm{n}}>20 \mathrm{~kg} / \mathrm{mol}\right)$. The red symbols refer to measured diffusion coefficients for low molecular weight $\left(M_{\mathrm{n}}=400 \mathrm{~g} / \mathrm{mol}\right)$ PEO homopolymers. In our study, we employ a hydroxylterminated PEO homopolymer, whereas Orädd et al. studied a dimethoxy-terminated PEO homopolymer. Data in figure are reproduced from Refs. 2 and 3.

\section{Analysis of electrochemical impedance spectroscopy (EIS) measurements}

When reporting conductivity data derived from EIS experiments, the lengthscale probed by the experiment is not immediately apparent. Because the interpretation of the data is dependent upon this lengthscale, we have worked to provide a reasonable estimate. A simple route to this estimate is through a calculation of the root mean squared (r.m.s.) displacement, given by

$$
l=\sqrt{6 D t}
$$

where $D$ is a diffusion coefficient and $t$ is the experimental time. For a case example, consider the r.m.s. displacement of ions in blends containing $\phi_{\mathrm{H}}=0.7$ as a function of temperature during EIS experiments. To perform this calculation at various temperatures, we need both $D(T)$ and $t(T)$. As stated in the Experimental Section, the conductivity of the sample was determined by extracting the value of the in-phase impedance, $Z^{\prime}$, at the high frequency plateau. Specifically, 
the value of $Z^{\prime}$ was chosen where $Z^{\prime \prime}$ was at a minimum. Thus, it is possible to calculate a time scale associated with the measurement by taking the inverse of the dielectric frequency, i.e. $t=$ $1 / f$. This time scale is shown at different temperatures in Figure S7a. Lines protruding from the symbols indicate the range of time scales represented by the width of the plateau (see Figure S8 for a visual example). From the PFG-NMR experiments (Figure S6), we expect that both the $\mathrm{Li}^{+}$ and $\mathrm{TFSI}^{-}$species in this study exhibit a diffusion coefficient of approximately $10^{-11} \mathrm{~m}^{2} / \mathrm{s}$ or greater at $75{ }^{\circ} \mathrm{C}$. The Vogel-Fulcher-Tammann-Hesse (VFTH) equation provides a method for calculating the viscosity of a blend at a given temperature and is given by: ${ }^{4}$

$$
\ln \eta=A+\frac{B}{T-T_{0}}
$$

where $A$ is an empirical constant, $T_{0}$ is the Vogel temperature, which is typically about $45^{\circ} \mathrm{C}$ below the $T_{\mathrm{g}}$, and $B$ describes the energetic barrier to, in this case, ion motion, just as it does in the VFT equation (Equation 2 in main text). $D \sim 1 / \eta$, so we can take the difference of the VFTH equation at two temperatures to yield

$$
\ln \eta-\ln \eta_{\mathrm{r}}=\ln \frac{\eta}{\eta_{\mathrm{r}}}=\ln \frac{D_{\mathrm{r}}}{D}=B\left(\frac{1}{T-T_{0}}-\frac{1}{T_{\mathrm{r}}-T_{0}}\right)
$$

where the subscript $r$ refers to the parameter at a reference temperature. For blends with $\phi_{\mathrm{H}}=0.7$, fits of the VFT equation to the conductivity of the ordered state gave a $B$ of $2600 \mathrm{~K}$ and $T_{0}$ of $115 \mathrm{~K}$. (Table S1). From the PFG-NMR results, we can use a value of $10^{-11} \mathrm{~m}^{2} / \mathrm{s}$ and $75^{\circ} \mathrm{C}$ for $D_{\mathrm{r}}$ and $T_{\mathrm{r}}$, respectively. This approximate route to determining diffusion coefficients was used to calculate the curve seen in Figure S6a. With the values for the diffusion coefficient thus calculated and the time scales extracted from the conductivity data, it was then possible to approximate the root mean squared displacement using Equation S1. These results are shown in Figure S7b, along with the domain spacings and conductivity data determined using SAXS and EIS experiments, respectively. 
(a)

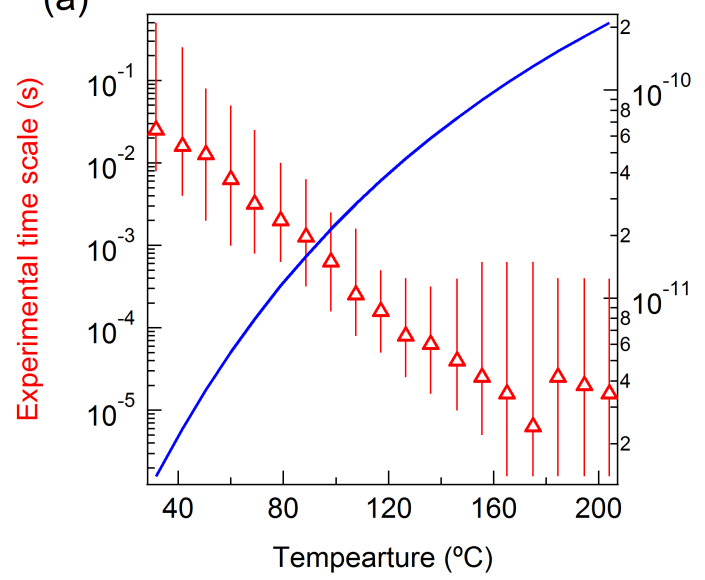

(b)

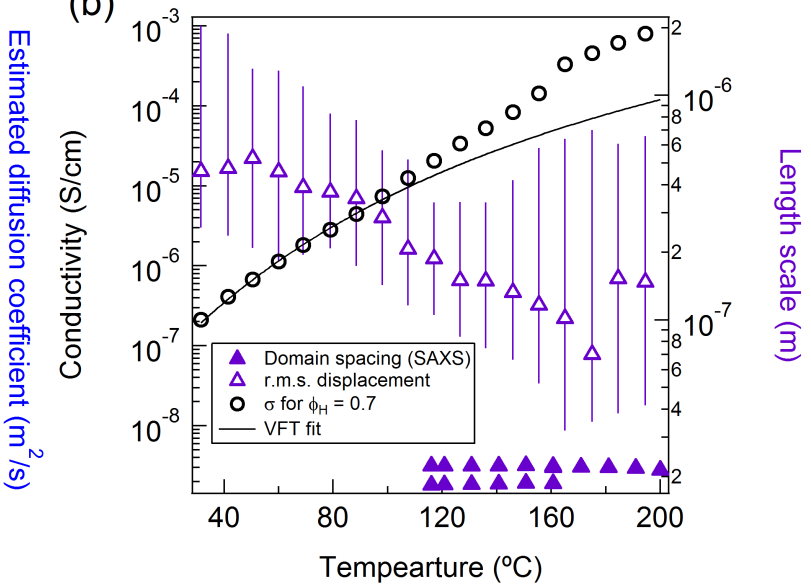

Figure S7. (a) Experimental time scales from EIS experiments and estimated diffusion coefficients for ions as a function of temperature. (b) Comparison of the estimated r.m.s. displacement of ions with the domain spacing from SAXS and conductivity data from EIS. The open triangles refer to the points at which $Z^{\prime \prime}$ was at a minimum, while the lines protruding from the triangles indicate the range of either time scales or length scales within the plateau region of the EIS spectra.

Figure S7b indicates that, throughout the entire temperature range probed, the r.m.s. displacements are about 3 to 10 times larger than the domain spacings of the blends. This indicates that the bulk resistance used to calculate conductivity considers the resistance ions experience when traversing over distances of multiple domain spacings. Furthermore, there is no correlation between the trend in the conductivity data with the r.m.s. displacement, thus strongly suggesting that the jump in conductivity is not related to the length scales probed by the experiment. 


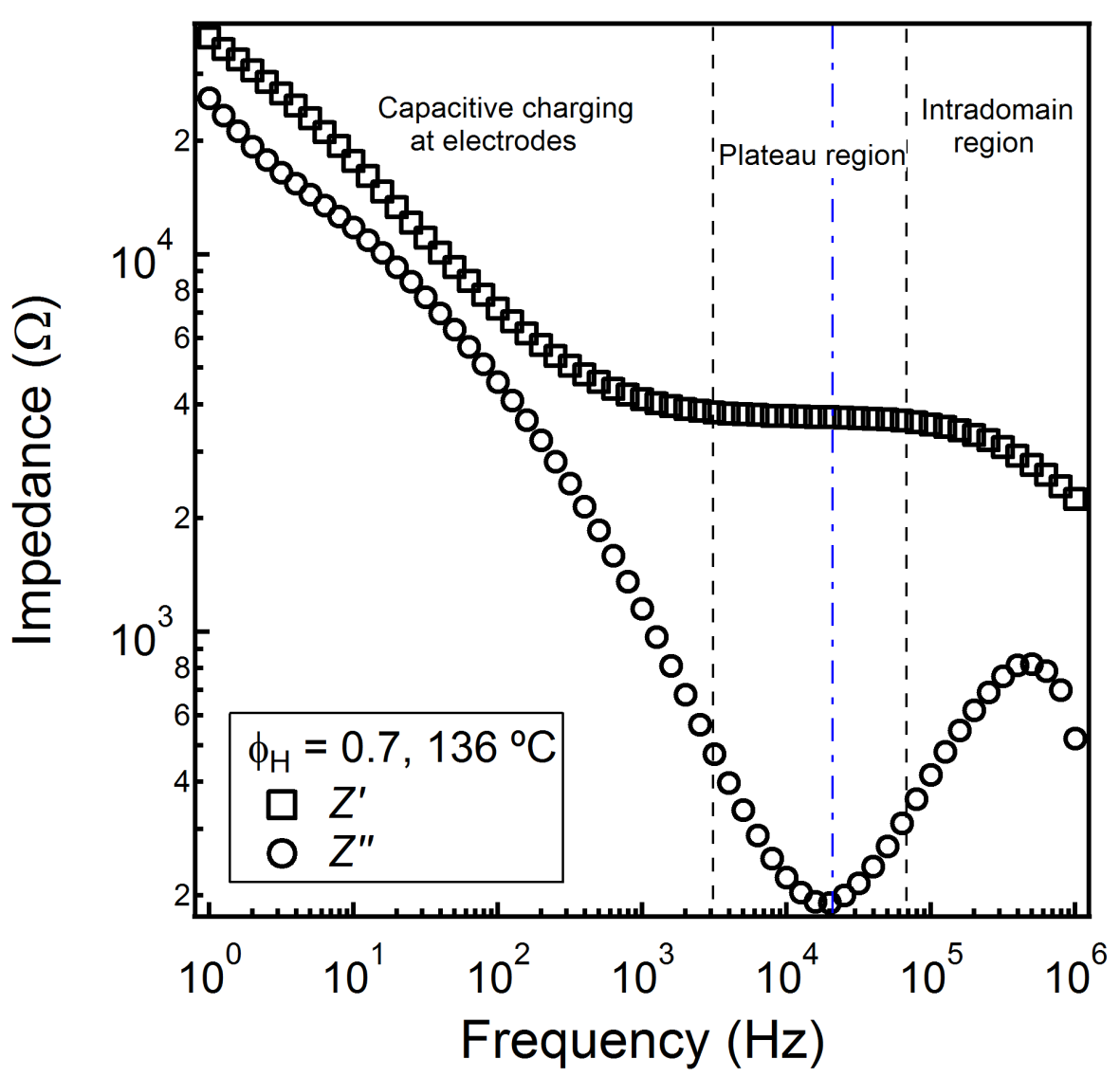

Figure S8. Representative EIS plot of $Z^{\prime}$ and $Z^{\prime \prime} v s$ dielectric frequency. The dashed lines represent the cutoffs of the high-frequency plateau region, while the dash-dot line indicates the frequency within the plateau region at which $Z^{\prime \prime}$ was minimized and, therefore where the bulk resistance of the sample was extracted. In the intradomain region at higher frequencies, the ions do not have enough time to traverse enough of the sample and thus experience a lower $Z^{\prime}$. At lower frequencies, ions have enough time to build up concentration gradients near the electrodes that lead to capacitive overpotentials, thus resulting in an increased $Z$ '. 


\section{References for Supporting Information}

1 Irwin, M. T.; Hickey, R. J.; Xie, S.; Bates, F. S.; Lodge, T. P. Lithium Salt-induced Microstructure and Ordering in Diblock Copolymer/Homopolymer Blends. Macromolecules 2016.

2 Orädd, G.; Edman, L.; Ferry, A. Diffusion: A comparison between liquid and solid polymer LiTFSI electrolytes. Solid State Ionics 2002, 152-153, 131-136.

3 Mullin, S. A.; Stone, G. M.; Panday, A.; Balsara, N. P. Salt Diffusion Coefficients in Block Copolymer Electrolytes. J. Electrochem. Soc. 2011, 158, A619.

4 Hiemenz, P. C.; Lodge, T. P. Polymer Chemistry, 2nd ed.; CRC Press: Boca Raton, FL, 2007. 